\title{
Operational solution to the nonlinear Klein-Gordon equation
}

\author{
G. Bengochea ${ }^{1, \dagger}$, L. Verde-Star ${ }^{1}$, M. Ortigueira ${ }^{2}$ \\ ${ }^{1}$ Departamento de Matemáticas, Universidad Autónoma Metropolitana, Iztapalapa, Apartado 55-534, Ciudad de \\ México, México. \\ ${ }^{2}$ CTS-UNINOVA/Department of Electrical Engineering, Faculdade de Ciéncias e Tecnologia da Universidade \\ Nova de Lisboa, Campus da FCT da UNL, Quinta da Torre 2825-149 Monte da Caparica, Portugal.
}

\begin{abstract}
We obtain solutions of the nonlinear Klein-Gordon equation using a novel algebraic operational method combined with the Adomian polynomial expansion of nonlinear functions. Our operational method does not use any integral transforms nor integration processes. We illustrate the application of our method by solving several examples and present numerical results that show the accuracy of the truncated series approximations to the solutions.
\end{abstract}

Keywords. Operational Calculus; Partial differential equations; Nonlinear Klein-Gordon equation.

MSC 2010. 44A 40,35A25.

\section{Introduction}

The nonlinear Klein-Gordon equation plays an important role in several fields of physics, such as quantum mechanics, general relativity and nonlinear optics, $[1,2]$. In the literature, there are numerous papers dealing with diverse methods to solve nonlinear Klein-Gordon equations such as the collocation method [3], the decomposition method [4], the homotopy method [5] and other methods [6, 7]. The nonlinear Klein-Gordon equation that we will study in this paper has the form

$$
\frac{\partial^{2} y(x, t)}{\partial t^{2}}-\Delta y(x, t)+b y(x, t)+g(y(x, t))=f(x, t)
$$

with $t \geq 0$, and initial conditions

$$
y(x, 0)=a_{0}(x), \quad \frac{\partial y}{\partial t}(x, 0)=a_{1}(x)
$$

where

$$
\Delta=\sum_{j=1}^{m} \frac{\partial^{2}}{\partial x_{j}^{2}},
$$

$x=\left(x_{1}, x_{2}, \ldots, x_{m}\right) \in \mathbb{R}^{m}, t \in(0, T], b$ is a real number, $g$ is a given nonlinear function, assumed to be analytic at zero, and $f$ is a known function. We study the one dimensional case $(m=1)$, but it is not difficult to see that the method can be applied in the general case.

The operational calculus that we will use in this paper is a particular instance of a general algebraic operational calculus introduced in [8]. Our operational calculus uses basic linear algebra tools to solve equations involving operators that generalize differential and difference operators. The algebraic setting is a field of generalized Laurent series with a multiplication, called algebraic convolution, and a modified shift operator that can be considered as a generalization of differentiation. Taking suitable concrete realizations of our operational calculus we can solve several kinds of differential and difference equations using the same procedure for all kinds of equations.

In this paper, we will use an algebraic convolution product that extends the multiplication formula $\left(t^{n} / n !\right) *$ $\left(t^{m} / m !\right)=t^{n+m} /(n+m)$ ! and coincides with the usual convolution defined by means of integrals. The concrete realization of the operational calculus that we will use allows us to solve linear and nonlinear differential equations, without using integral transforms or computing integrals. Since our operational methods are based on simple linear algebra they are simpler than those based on the Mikusiński operational calculus [9], and can be used to provide a rigorous foundation of the main results of Heaviside's operational calculus. Several applications of our methods can be found in [10-13], and some variations in [14, 15].

\footnotetext{
${ }^{\dagger}$ Corresponding author, E-mail: g.bengochea@xanum.uam.mx
} 
The nonlinear term in (1) will be handled by means of the Adomian polynomials [16], which we calculate using expansions in Taylor series. Combining our methods with the Adomian polynomial expansions we solve (1) with a purely operational approach.

The paper is organized as follows. Section 2 contains an introduction to the operational calculus introduced in [8], and the procedure to calculate the Adomian polynomials. In Section 3 we solve (1) using the tools introduced in Section 2. In Section 4 we show how the method is applied to several equations that have been considered in research papers related with the subject. Finally, some conclusions are presented in Section 5.

\section{Algebraic setting and preliminary results}

\subsection{Operational Calculus}

In this section we present a brief description of the algebraic setting and the basic properties of the general operational calculus introduced in the paper [8], where the reader can find a detailed presentation and the proofs of the statements that we summarize next.

Let $\left\{p_{k}: k \in \mathbb{Z}\right\}$ be a group with the multiplication defined by $p_{k} p_{n}=p_{k+n}$, for $k, n \in \mathbb{Z}$. Let $\mathcal{F}$ be the set of all the formal series of the form

$$
a=\sum_{k \in \mathbb{Z}} a_{k} p_{k}
$$

where $a_{k}$ is a complex number for each $k \in \mathbb{Z}$ and, either, all the $a_{k}$ are equal to zero, or there exists an integer $v(a)$ such that $a_{k}=0$ whenever $k<v(a)$ and $a_{v(a)} \neq 0$. In the first case we write $a=0$ and define $v(0)=\infty$. Addition in $\mathcal{F}$ and multiplication by complex numbers are defined in the usual way. The multiplication in $\mathcal{F}$ is defined by extending the multiplication of the group $\left\{p_{k}: k \in \mathbb{Z}\right\}$ as follows. If $a=\sum a_{k} p_{k}$ and $b=\sum b_{k} p_{k}$ are elements of $\mathcal{F}$ then $a b=\sum c_{n} p_{n}$, where the coefficients $c_{n}$ are given by

$$
c_{n}=\sum_{v(a) \leq k \leq n-v(b)} a_{k} b_{n-k} .
$$

This multiplication in $\mathcal{F}$ is associative and commutative and $p_{0}$ is its unit element. With this multiplication $\mathcal{F}$ is a field. We define the series

$$
e_{x, k}=\sum_{n \geq k}\left(\begin{array}{l}
n \\
k
\end{array}\right) x^{n-k} p_{n}, \quad x \in \mathbb{C}, k \geq 0 .
$$

These elements of $\mathcal{F}$ are called generalized geometric series and satisfy

$$
e_{x, k}\left(p_{-k}\left(p_{0}-x p_{1}\right)^{k+1}\right)=p_{0} .
$$

In particular, when $k=0$ we have

$$
e_{x, 0}\left(p_{0}-x p_{1}\right)=p_{0}
$$

If $x \neq y$ then

$$
p_{1} e_{x, m} e_{y, n}=\sum_{i=0}^{m} \frac{\left(\begin{array}{c}
n+i \\
i
\end{array}\right)(-1)^{i} e_{x, m-i}}{(x-y)^{1+n+i}}+\sum_{j=0}^{n} \frac{\left(\begin{array}{c}
m+j \\
j
\end{array}\right)(-1)^{j} e_{y, n-j}}{(y-x)^{1+m+j}} .
$$

In particular, if $m=n=0$ then we have

$$
p_{1} e_{x, 0} e_{y, 0}=\frac{e_{x, 0}-e_{y, 0}}{x-y}, \quad x \neq y .
$$

The linear operator $L$ on $\mathcal{F}$ is defined by $L p_{k}=p_{k-1}$ for $k \neq 0$, and $L p_{0}=0$, and it is called the modified left shift. As an example, if $a=\sum_{i \geq 0} y_{i} p_{i}$, then $L a=\sum_{i \geq 0} y_{i+1} p_{i}$. One important property of the operator $L$ is

$$
L^{k}=p_{-k}\left(p_{0} I-P_{0}-P_{1}-P_{2}-\cdots-P_{k-1}\right)
$$

where $I$ is the identity operator and $P_{n}$ is the projection on the subspace generated by $p_{n}$, that is $P_{n} a=y_{n} p_{n}$. See [8, p. 333]. 
In order to apply our operational method to nonlinear Klein-Gordon differential equations we will use the concrete realization of the field $\mathcal{F}$ that has $p_{k}=t^{k} / k$ !, for $k \in \mathbb{Z}$, where $t$ is a real or complex variable and $k$ ! is defined for negative values of $k$ by

$$
k !=\frac{(-1)^{-k-1}}{(-k-1) !}, \quad k<0 .
$$

The modified left shift in this concrete realization is $L=\partial / \partial t=D_{t}$, and the generalized geometric series become the basic exponential polynomials

$$
e_{x, k}=\frac{t^{k}}{k !} \exp (x t), \quad x \in \mathbb{C}, \quad k \geq 0 .
$$

Let us note that the generalized geometric series are convergent for all $x$ and $t$ in $\mathbb{C}$.

If instead of taking $p_{k}=t^{k} / k$ ! we take $p_{k}$ as a normalized Hermite polynomial of degree $k$ we obtain another concrete realization of the operational calculus that can be used to solve differential equations of the kind that we consider in this paper.

\section{$2.2 \quad$ Adomian polynomials}

The Adomian polynomials and decomposition methods [16] are a very important tool for the solution of many kinds of equations, including nonlinear differential equations. The general theory and convergence properties of the Adomian decomposition methods have been studied in [17-19], but, in many applications the convergence properties depend on the particular functions involved in the equations. In the examples that we consider in this paper the domain of convergence of the series that represent the solutions is easily determined.

Using the Adomian polynomials we can write a nonlinear term $g(y)$ as a power series. In this paper, we calculate the Adomian polynomials by expanding the nonlinear term $g(y)$ in a Taylor series about the point $y_{0}$, that is

$$
g(y)=\left.\sum_{k=0}^{\infty} \frac{1}{k !} \frac{d^{k}}{d y^{k}} g(y)\right|_{y=y_{0}}\left(y-y_{0}\right)^{k},
$$

where $y=\sum y_{k} t^{k} / k$ !. After some algebraic manipulation and grouping terms that correspond to the same monomial we obtain a series

$$
g(y)=\sum_{k=0}^{\infty} A_{k} \frac{t^{k}}{k !}=\sum_{k=0}^{\infty} A_{k} p_{k} .
$$

where the $A_{k}$ are the Adomian polynomials.

\section{Operational solution of nonlinear Klein-Gordon equations}

In this Section we will solve (1) using the theory introduced in Section 2. We will take $\Delta=\partial^{2} / \partial x^{2}$ and suppose that $f(x, t)$ is of the form

$$
f(x, t)=\sum_{k=0}^{n} f_{1, k}(x) f_{2, k}(t),
$$

where the functions $f_{1, k}$ and $f_{2, k}$ have a convergent Taylor series representation in a neighborhood of zero and

$$
f_{2, k}(t)=\sum_{j=0}^{\infty} b_{j, k} p_{j}, \quad 0 \leq k \leq n .
$$

Suppose that the solution of (1) can be expressed in the form $y(x, t)=\sum y_{k}(x) t^{k} / k$ !, for some sequence of functions $y_{k}(x)$. Using the concrete realization of the operational method with $p_{k}=t^{k} / k$ ! and $L=\partial / \partial t=D_{t}$ we can rewrite (1) as

$$
L^{2} y+b y=\sum_{k=0}^{\infty} D_{x}^{2}\left(y_{k}(x)\right) p_{k}-g(y)+\sum_{k=0}^{n} f_{1, k}(x) \sum_{j=0}^{\infty} b_{j, k} p_{j}
$$

From (4) we have that $L^{2}=p_{-2}\left(p_{0} I-P_{0}-P_{1}\right)$ and therefore the previous equation becomes

$$
\left(p_{-2}+b p_{0}\right) y=y_{0}(x) p_{-2}+y_{1}(x) p_{-1}+\sum_{k=0}^{\infty} D_{x}^{2}\left(y_{k}(x)\right) p_{k}-g(y)+\sum_{k=0}^{n} f_{1, k}(x) \sum_{j=0}^{\infty} b_{j, k} p_{j} .
$$


From (5) we write $g(y)$ in terms of the Adomian polynomials as $\sum_{k=0}^{\infty} A_{k} p_{k}$. Also observe that we have the factorization $\left(p_{-2}+b p_{0}\right)=\left(p_{-1}+i \sqrt{b} p_{0}\right)\left(p_{-1}-i \sqrt{b} p_{0}\right)$ and that, by $(2)$, the multiplicative inverse of $\left(p_{-2}+b p_{0}\right)$ in the field $\mathcal{F}$ is $p_{2} e_{i \sqrt{b}, 0} e_{-i \sqrt{b}, 0}$. Therefore

$$
\sum_{k=0}^{\infty} y_{k}(x) p_{k}=e_{i \sqrt{b}, 0} e_{-i \sqrt{b}, 0}\left(y_{0}(x) p_{0}+y_{1}(x) p_{1}+\sum_{k=0}^{\infty}\left(D_{x}^{2}\left(y_{k}(x)\right)-A_{k}\right) p_{k+2}+\sum_{k=0}^{n} f_{1, k}(x) \sum_{j=0}^{\infty} b_{j, k} p_{j+2}\right) .
$$

Performing the operations in the right-hand side of (6) we obtain a series in terms of the $p_{k}$ which begins with $p_{0}$. Consider that $y_{0}(x)$ and $y_{1}(x)$ are the initial conditions $a_{0}(x)$ and $a_{1}(x)$, respectively. Then we equate the coefficients of $p_{k}$ in both sides of the equation and obtain recursively the solution $y_{k}(x)$. Finally, we replace $p_{k}$ by $t^{k} / k$ ! and obtain the solution as a function of $t$ and $x$. In the next Section we will solve in detail several examples.

Remark 1 It is worth mentioning that the operations in (6) can be simplified using the multiplication formula (3).

\section{Implementation of the operational method}

Our first example is a linear non-homogeneous equation.

Example 2 Consider the non-homogeneous Klein-Gordon equation of the form

$$
\frac{\partial^{2} y}{\partial t^{2}}-\frac{\partial^{2} y}{\partial x^{2}}-2 y=-2 \sin x \sin t, \quad t \geq 0,
$$

with initial conditions

$$
y(x, 0)=\mathrm{e}^{-a x}, \quad \frac{\partial y}{\partial t}(x, 0)=0, \quad a \geq 0
$$

Equation (7) can be written as

$$
\left(D_{t}^{2}-2 I\right) y=D_{x}^{2} y-2 \sin x \sin t
$$

where $D_{t}$ and $D_{x}$ denote the derivatives with respect to $t$ and $x$, respectively, and $I$ is the identity operator. Suppose that the solution can be written in the form

$$
y(x, t)=\sum_{k=0}^{\infty} y_{k}(x) \frac{t^{k}}{k !}
$$

for some sequence of functions $y_{k}(x)$. Observe that

$$
D_{x}^{2} y=\sum_{k=0}^{\infty} D_{x}^{2}\left(y_{k}(x)\right) \frac{t^{k}}{k !}
$$

and using the Taylor series expansion at zero of $\sin (t)$ we obtain

$$
\left(D_{t}^{2}-2 I\right) y(x, t)=\sum_{k=0}^{\infty} D_{x}^{2}\left(y_{k}(x)\right) \frac{t^{k}}{k !}-2 \sin x \sum_{k=0}^{\infty}(-1)^{k} \frac{t^{2 k+1}}{(2 k+1)} .
$$

Let $p_{k}=t^{k} / k$, for $k \in \mathbb{Z}$, and $L=D_{t}$. Since, by (4), we have $L^{2}=p_{-2}\left(p_{0} I-P_{0}-P_{1}\right)$, equation (8) can be expressed in the form

$$
\left(p_{-2}\left(p_{0} I-P_{0}-P_{1}\right)-2 p_{0} I\right) y(x, t)=\sum_{k=0}^{\infty} D_{x}^{2}\left(y_{k}(x)\right) p_{k}-2 \sin x \sum_{k=0}^{\infty}(-1)^{k} p_{2 k+1}
$$

After some algebraic manipulations and using the fact that $P_{k} y(x, t)=y_{k}(x) p_{k}$, we get

$$
\left(p_{0}-\sqrt{2} p_{1}\right)\left(p_{0}+\sqrt{2} p_{1}\right) y(x, t)=y_{0}(x) p_{0}+y_{1}(x) p_{1}+\sum_{k=0}^{\infty} D_{x}^{2}\left(y_{k}(x)\right) p_{k+2}-2 \sin x \sum_{k=0}^{\infty}(-1)^{k} p_{2 k+3} .
$$


From (2) we see that $e_{\sqrt{2}, 0} e_{-\sqrt{2}, 0}$ is the multiplicative inverse of $\left(p_{0}-\sqrt{2} p_{1}\right)\left(p_{0}+\sqrt{2} p_{1}\right)$ in $\mathcal{F}$. Hence

$$
y(x, t)=e_{\sqrt{2}, 0} e_{-\sqrt{2}, 0}\left(y_{0}(x) p_{0}+y_{1}(x) p_{1}+\sum_{k=0}^{\infty} D_{x}^{2}\left(y_{k}(x)\right) p_{k+2}-2 \sin x \sum_{k=0}^{\infty}(-1)^{k} p_{2 k+3}\right) .
$$

From (3) it is clear that

$$
e_{\sqrt{2}, 0} e_{-\sqrt{2}, 0}=\frac{1}{2 \sqrt{2}} \sum_{k=1}^{\infty}\left((\sqrt{2})^{k}-(-\sqrt{2})^{k}\right) p_{k-1}=\sum_{k=0}^{\infty} 2^{k} p_{2 k}
$$

In order to simplify the notation we will write $y_{k}$ instead of $y_{k}(x)$ and $y$ instead of $y(x, t)$. Therefore we have

$$
\begin{aligned}
\sum_{k=0}^{\infty} y_{k} p_{k} & =\left(\sum_{k=0}^{\infty} 2^{k} p_{2 k}\right)\left(y_{0} p_{0}+y_{1} p_{1}+\sum_{k=0}^{\infty} D_{x}^{2}\left(y_{k}\right) p_{k+2}-2 \sin x \sum_{k=0}^{\infty}(-1)^{k} p_{2 k+3}\right) \\
& =y_{0} p_{0}+y_{1} p_{1}+\left(y_{0}+D_{x}^{2}\left(y_{0}\right)\right) p_{2}+\left(2 y_{1}-2 \sin x+D_{x}^{2}\left(y_{1}\right)\right) p_{3}+\left(4 y_{0}+D_{x}^{2}\left(y_{2}+2 y_{0}\right)\right) p_{4}+\cdots .
\end{aligned}
$$

Equating corresponding coefficients of $p_{k}$ in both sides of the last equation we get

$$
\begin{aligned}
& y_{0}=y_{0} \\
& y_{1}=y_{1} \\
& y_{2}=y_{0}+D_{x}^{2}\left(y_{0}\right) \\
& y_{3}=2 y_{1}-2 \sin x+D_{x}^{2}\left(y_{1}\right) \\
& y_{4}=4 y_{0}+D_{x}^{2}\left(y_{2}+2 y_{0}\right) \\
& \vdots=\quad \vdots
\end{aligned}
$$

The initial conditions give us $y_{0}=\mathrm{e}^{-a x}$ and $y_{1}=0$. Then the previous equations become $y_{2}=\left(a^{2}-2\right) \mathrm{e}^{-a x}, y_{3}=$ $-\left(3^{1}-1\right) \sin x, y_{4}=\left(a^{2}-2\right)^{2} \mathrm{e}^{-a x}, y_{5}=\left(3^{2}-1\right) \sin x, \ldots$ Therefore the solution is given by

$$
y=\mathrm{e}^{-a x} \sum_{k=0}^{\infty}\left(a^{2}-2\right)^{k} p_{2 k}+\sin (x) \sum_{k=1}^{\infty}(-1)^{k}\left(3^{k}-1\right) p_{2 k+1},
$$

and then, writing the $p_{k}$ in terms of $t$ we obtain

$$
y(x, t)=\mathrm{e}^{-a x} \sum_{k=0}^{\infty}\left(a^{2}-2\right)^{k} \frac{t^{2 k}}{(2 k) !}+\sin (x) \sum_{k=0}^{\infty}(-1)^{k}\left(3^{k}-1\right) \frac{t^{2 k+1}}{(2 k+1) !} .
$$

If we define $A^{2}=a^{2}-2$ and compute the sums we get

$$
\begin{aligned}
y(x, t) & =\mathrm{e}^{-a x} \sum_{k=0}^{\infty} \frac{(A t)^{2 k}}{(2 k) !}+\sin (x)\left(\sum_{k=0}^{\infty}(-1)^{k}\left(3^{k}\right) \frac{t^{2 k+1}}{(2 k+1) !}-\sum_{k=0}^{\infty}(-1)^{k} \frac{t^{2 k+1}}{(2 k+1) !}\right) \\
& =\mathrm{e}^{-a x} \sum_{k=0}^{\infty} \frac{(A t)^{2 k}}{(2 k) !}+\sin (x)\left(\frac{1}{\sqrt{3}} \sum_{k=0}^{\infty}(-1)^{k} \frac{(\sqrt{3} t)^{2 k+1}}{(2 k+1) !}-\sum_{k=0}^{\infty}(-1)^{k} \frac{t^{2 k+1}}{(2 k+1) !}\right) \\
& =\mathrm{e}^{-a x} \cos \left(\left(a^{2}-2\right) t\right)+\frac{\sin (x)}{\sqrt{3}} \sin (\sqrt{3} t)-\sin (x) \sin (t) .
\end{aligned}
$$

Remark 3 If we change the initial conditions in Example 2 and consider instead

$$
y(x, 0)=0, \quad \frac{\partial y}{\partial t}(x, 0)=\sin (x) .
$$

we obtain $y_{0}=0$ and $y_{1}=\sin (x)$, and recursively $y_{2}=0, y_{3}=-\sin (x), y_{4}=0, y_{5}=\sin (x), \ldots$ The solution in terms of the concrete realization is given by

$$
y(x, t)=\sin x\left(t-\frac{t^{3}}{3 !}+\frac{t^{5}}{5 !}-\cdots\right)=\sin x \sin t
$$


Our solution coincides with the solution obtained in [4]. The series $t-\frac{t^{3}}{3 !}+\frac{t^{5}}{5 !}-\cdots$ is convergent for all real values of $t$ since it is the expansion in Taylor series of $\sin (t)$. Therefore our solution converges for all real values of $t$. Table 1 shows the absolute error between our truncated series solution (15 terms) and the closed form solution.

Example 4 Now, consider the nonlinear Klein-Gordon equation of the form

$$
\frac{\partial^{2} y}{\partial t^{2}}-\frac{\partial^{2} y}{\partial x^{2}}+y^{2}=-x \cos (t)+x^{2} \cos ^{2}(t)
$$

with initial conditions

$$
y(x, 0)=x, \quad \frac{\partial y}{\partial t}(x, 0)=0, \quad-1 \leq x \leq 1 .
$$

Equation (9) can be written as

$$
D_{t}^{2} y=D_{x}^{2} y-y^{2}-x \cos (t)+x^{2} \cos ^{2}(t) .
$$

Let us suppose that the solution has the form

$$
y=\sum_{k=0}^{\infty} y_{k} \frac{t^{k}}{k !}
$$

where the $y_{k}$ are functions of $x$. Then we have

$$
D_{x}^{2} y=\sum_{k=0}^{\infty} D_{x}^{2}\left(y_{k}\right) \frac{t^{k}}{k !}
$$

The nonlinear term $y^{2}$ can be expanded as a Taylor series about the point $y_{0}$ as follows

$$
\begin{aligned}
y^{2} & =y_{0}^{2}+2 y_{0}\left(y-y_{0}\right)+\left(y-y_{0}\right)^{2} \\
& =y_{0}^{2}+2 y_{0} y_{1} t+\left(2 y_{0} y_{2}+2 y_{1}^{2}\right) \frac{t^{2}}{2 !}+\left(6 y_{1} y_{2}+2 y_{0} y_{3}\right) \frac{t^{3}}{3 !}+\cdots
\end{aligned}
$$

and the function $-x \cos (t)+x^{2} \cos ^{2}(t)$ is expressed as the convergent series

$$
-x \cos (t)+x^{2} \cos ^{2}(t)=-x+x^{2}+\left(x-2 x^{2}\right) \frac{t^{2}}{2 !}+\left(-x+8 x^{2}\right) \frac{t^{4}}{4 !}+\left(x-32 x^{2}\right) \frac{t^{6}}{6 !}+\cdots
$$

Following the same procedure used in Example 2, we write (9) in terms of the $p_{k}$ 's as

$$
\begin{gathered}
p_{-2} y=y_{0} p_{-2}+y_{1} p_{-1}+\sum_{k=0}^{\infty} D_{x}^{2}\left(y_{k}\right) p_{k}-y_{0}^{2} p_{0}-2 y_{0} y_{1} p_{1}-\left(2 y_{0} y_{2}+2 y_{1}^{2}\right) p_{2}-\cdots+\left(-x+x^{2}\right) p_{0}+ \\
\sum_{i=1}^{\infty}(-1)^{i}\left(-x+2^{2 i-1} x^{2}\right) p_{2 i} .
\end{gathered}
$$

Multiplying both sides of the equation by $p_{2}$, which is the multiplicative inverse of $p_{-2}$, we obtain

$$
\begin{aligned}
& \sum_{k=0}^{\infty} y_{k} p_{k}=p_{2}\left(y_{0} p_{-2}+y_{1} p_{-1}+\sum_{k=0}^{\infty} D_{x}^{2}\left(y_{k}\right) p_{k}-y_{0}^{2} p_{0}-2 y_{0} y_{1} p_{1}-\cdots+\left(-x+x^{2}\right) p_{0}+\right. \\
&\left.\sum_{i=1}^{\infty}(-1)^{i}\left(-x+2^{2 i-1} x^{2}\right) p_{2 i}\right) \\
&=y_{0} p_{0}+y_{1} p_{1}-\left(x-x^{2}+y_{0}^{2}-D_{x}^{2}\left(y_{0}\right)\right) p_{2}-\left(2 y_{0} y_{1}-D_{x}^{2}\left(y_{1}\right)\right) p_{3}- \\
&\left(-x+2 x^{2}+2 y_{0} y_{2}-D_{x}^{2}\left(y_{2}\right)\right) p_{4}+\cdots
\end{aligned}
$$

Equating corresponding coefficients of the $p_{k}$ in both side of the equation we get

$$
\begin{aligned}
& y_{0}=y_{0} \\
& y_{1}=y_{1} \\
& y_{2}=-\left(x-x^{2}+y_{0}^{2}-D_{x}^{2}\left(y_{0}\right)\right) \\
& y_{3}=-\left(2 y_{0} y_{1}-D_{x}^{2}\left(y_{1}\right)\right) \\
& y_{4}=-\left(-x+2 x^{2}+2 y_{0} y_{2}-D_{x}^{2}\left(y_{2}\right)\right) \\
& \vdots=\vdots
\end{aligned}
$$


The initial conditions give us $y_{0}=x$ and $y_{1}=0$, and by forward substitutions we obtain $y_{2}=-x, y_{3}=0, y_{4}=$ $x, y_{5}=0, \ldots$. Therefore the solution is given by $\sum_{k \geq 0}(-1)^{k} x p_{2 k}$, and this is equivalent to

$$
y(x, t)=x\left(1-\frac{t^{2}}{2 !}+\frac{t^{4}}{4 !}-\cdots\right)=x \cos (t)
$$

Our solution coincides with the solution presented in [20]. The series $1-\frac{t^{2}}{2 !}+\frac{t^{4}}{4 !}-\cdots$ is convergent for all real values of $t$ since it is the expansion in Taylor series of $\cos (t)$. Therefore our solution converges for all real values of $t$. Table 2 shows the absolute error between our approximation to the solution by a truncated series and the closed form solution.

Example 5 Consider now the nonlinear Klein-Gordon equation

$$
\frac{\partial^{2} y}{\partial t^{2}}+\alpha \frac{\partial^{2} y}{\partial x^{2}}+\beta y+\gamma y^{3}=0
$$

with initial conditions

$$
y(x, 0)=B \tan (K x), \quad \frac{\partial y}{\partial t}(x, 0)=B c K \sec ^{2}(K x),
$$

where $L_{0} \leq x \leq L_{1}, t_{0} \leq t$, and $c, \alpha, \beta, \gamma$ are constants, and $B=\sqrt{\beta / \gamma}, K=\sqrt{-\beta /\left(2\left(\alpha+c^{2}\right)\right)}$. Equation (10) can be written as

$$
\left(D_{t}^{2}+\beta I\right) y=-\alpha D_{x}^{2} y-\gamma y^{3},
$$

As in the previous examples we suppose that

$$
y=\sum_{k=0}^{\infty} y_{k} \frac{t^{k}}{k !},
$$

and

$$
D_{x}^{2} y=\sum_{k=0}^{\infty} D_{x}^{2}\left(y_{k}\right) \frac{t^{k}}{k !},
$$

Expanding the nonlinear term $y^{3}$ as a Taylor series about the point $y_{0}$, we get

$$
\begin{aligned}
y^{3} & =y_{0}^{3}+3 y_{0}^{2}\left(y-y_{0}\right)+3 y_{0}\left(y-y_{0}\right)^{2}+\left(y-y_{0}\right)^{3} \\
& =y_{0}^{3}+3 y_{0}^{2} y_{1} t+\left(3 y_{0}^{2} y_{2}+6 y_{0} y_{1}^{2}\right) \frac{t^{2}}{2 !}+\left(3 y_{0}^{2} y_{3}+18 y_{0} y_{1} y_{2}+6 y_{1}^{3}\right) \frac{t^{3}}{3 !}+\cdots
\end{aligned}
$$

Following the same procedure that we used in Examples 2 and 4, we write (10) in terms of the $p_{k}$ 's as

$$
\left(p_{0}+d i p_{1}\right)\left(p_{0}-d i p_{1}\right) y=y_{0} p_{0}+y_{1} p_{1}-\alpha \sum_{k=0}^{\infty} D_{x}^{2}\left(y_{k}\right) p_{k}-\gamma\left(y_{0}^{3} p_{0}+3 y_{0}^{2} y_{1} p_{1}+\left(3 y_{0}^{2} y_{2}+6 y_{0} y_{1}^{2}\right) p_{2}+\cdots\right),
$$

where $d=\sqrt{\beta}$. Since the product $e_{-d i, 0} e_{d i, 0}$ is the multiplicative inverse of $\left(p_{0}+d i p_{1}\right)\left(p_{0}-d i p_{1}\right)$ in the field $\mathcal{F}$, we have

$$
\begin{aligned}
\sum_{k=0}^{\infty} y_{k} p_{k} & =e_{-d i, 0} e_{d i, 0}\left(y_{0} p_{0}+y_{1} p_{1}-\alpha \sum_{k=0}^{\infty} D_{x}^{2}\left(y_{k}\right) p_{k}-\gamma\left(y_{0}^{3} p_{0}+3 y_{0}^{2} y_{1} p_{1}+\left(3 y_{0}^{2} y_{2}+6 y_{0} y_{1}^{2}\right) p_{2}+\cdots\right)\right) \\
& =y_{0} p_{0}+y_{1} p_{1}+\left(-\gamma y_{0}^{3}-d^{2} y_{0}-\alpha D_{x}^{2}\left(y_{0}\right)\right) p_{2}+\left(-3 \gamma y_{0}^{2} y_{1}-d^{2} y_{1}-\alpha D_{x}^{2}\left(y_{1}\right)\right) p_{3}+\cdots
\end{aligned}
$$

Equating coefficients we get

$$
\begin{aligned}
& y_{0}=y_{0} \\
& y_{1}=y_{1} \\
& y_{2}=-\gamma y_{0}^{3}-d^{2} y_{0}-\alpha D_{x}^{2}\left(y_{0}\right) \\
& y_{3}=-3 \gamma y_{0}^{2} y_{1}-d^{2} y_{1}-\alpha D_{x}^{2}\left(y_{1}\right) \\
& \vdots=\vdots
\end{aligned}
$$


The given initial conditions yield $y_{0}=B \tan (K x)$ and $y_{1}=B c K \sec ^{2}(K x)$, and from the previous equations we obtain

$$
\begin{gathered}
y_{2}=-\gamma B^{3} \tan ^{3}(K x)-d^{2} B \tan (K x)-2 \alpha B K^{2} \tan (K x)\left(1+\tan ^{2}(K x)\right) \\
y_{3}=-3 \gamma B^{3} c K \tan ^{2}(K x) \sec ^{2}(K x)-d^{2} B c K \sec ^{2}(K x)-\alpha\left(4 B c K^{3} \sec ^{2}(K x) \tan ^{2}(K x)+\right. \\
\left.2 B c K^{3} \sec ^{2}(K x)\left(1+\tan ^{2}(K x)\right)\right) \\
y_{4}=\frac{B \sin (K x)}{\cos ^{5}(K x)}\left(3 \gamma^{2} B^{4} \cos ^{4}(K x)-4 \gamma d^{2} B^{2} \cos ^{4}(K x)-6 \gamma^{2} B^{4} \cos ^{2}(K x)-\right. \\
12 \gamma \alpha B^{2} K^{2} \cos ^{2}(K x)-8 \alpha^{2} K^{4} \cos ^{2}(K x)+d^{4} \cos ^{4}(K x)-6 \gamma c^{2} B^{2} K^{2}+ \\
\left.4 \gamma d^{2} B^{2} \cos ^{2}(K x)+4 \alpha d^{2} K^{2} \cos ^{2}(K x)+3 B^{4} \gamma^{2}+18 \gamma \alpha B^{2} K^{2}+24 K^{4} \alpha^{2}\right)
\end{gathered}
$$

Our series $\sum y_{k} t^{k} / k !$ with the coefficients described in (11) is the Taylor series, around $t=0$, of $y=B \tan (K(x+c t))$, which is the exact solution of (10), see [21]. The series obtained by our method is convergent for all real values of $t$ between $-\pi / 2$ and $\pi / 2$ since it is the expansion in Taylor series of $y=B \tan (K(x+c t))$. Table 3 shows the absolute error between our solution and the exact solution when we take $B=0.816497, K=0.426401$ and $c=0.5$.

\section{Conclusions}

In the examples presented in the previous section we have shown how our operational method is applied to obtain solutions of Klein-Gordon equations. In all the examples our solutions coincide with the ones obtained by other authors using diverse methods. The main difference between our method and the usual ones is that we do not require the computation of integrals, and we do not use any integral transforms. In addition, since the computational parts of our method are mainly operations with power series and differentiation, they can be easily done using symbolic computational software packages, such as Maple and Mathematica. The numerical results presented in the solved examples show that the values computed by evaluation of truncations of the series representations produce good approximations of the exact solutions. This fact indicates that we can expect to obtain good approximations to the solutions of problems for which the exact solution is not known.

Our operational methods can also be used to solve other kinds of differential and difference equations, including fractional differential equations, with several different definitions of the fractional differentiation operators. The

procedures used for all kinds of equations are essentially the same and do not depend on particular properties of the elements $p_{k}$ and the operator $L$ of the concrete realization used in each case.

\section{Acknowledgments}

The research reported in this paper was partially supported by grant SEP-CONACYT 220603. The first author was supported by SEP-PRODEP through the project UAM-PTC-630. The third author was supported by Portuguese National Funds through the FCT Foundation for Science and Technology under the project PEstUID/EEA/00066/2013. ...

\section{References}

[1] E. Zauderer, Partial differential equations of applied mathematics. John Wiley \& Sons, New Jersey (2006).

[2] R. Dodd, J. Eilbeck, J. Gibbon, and H. Morris, Solitons and nonlinear wave equations. Academic Press, Inc. Harcourt Brace Jovanovich, Publishers, London, New York (1982).

[3] R. Mittal and R. Bhatia, Int. J. Comput. Math. 92 (2015) 2139.

[4] E. Deeba and S. Khuri, J. Comput. Phys. 124 (1996) 442.

[5] D. Kumar, J. Singh, S. Kumar, et al., Alexandria Eng. J. 53 (2014) 469.

[6] H. Dong-mei, Z. Guo-liang, and L. Zhang, Math. Probl. Eng. 2015 (2015) 1. 
[7] N. Hayashi and P. Naumkin, Z. Angew. Math. Phys. 59 (2008) 1002.

[8] G. Bengochea and L. Verde-Star, Adv. in Appl. Math. 47 (2011) 330.

[9] J. Mikusiński. Operational Calculus, Pergamon Press, Oxford-Warszawa (1959).

[10] G. Bengochea, Appl. Math. Lett., 32 (2014) 48.

[11] G. Bengochea and L. Verde-Star, Math. Meth. Appl. Sci. 38 (2015) 4630.

[12] G. Bengochea, Appl. Math. Comput. 232 (2014) 424.

[13] G. Bengochea, Fract. Calc. Appl. Anal. 18 (2015) 1201.

[14] G. Bengochea and M. Ortigueira, J. Appl. Anal. 22 (2016) 131.

[15] G. Bengochea and M. Ortigueira, Int. J. Dyn. Control 5 (2017) 61.

[16] G. Adomian, Math. Comput. Model. 13 (1990) 17.

[17] Y. Cherruault and G. Adomian, Math. Comput. Model. 18 (1993) 103.

[18] G. Guellal and Y. Cherruault, Int. J. Bio-Med. Comput. 36 (1994) 223.

[19] M. Hosseini and H. Nasabzadeh, Appl. Math.Comput. 182 (2006) 536.

[20] P. Guo, K. Liew, and P. Zhu, Appl. Math. Model. 39 (2015) 2917.

[21] D. Kaya and S. El-Sayed, Appl. Math. Comput. 156 (2004) 341.

\begin{tabular}{|c||c|c|c|c|c|c|}
\hline \multicolumn{1}{|c|}{$x$} & 0.5 & 1 & 1.5 & 2 & 2.5 & 3 \\
\hline \hline 0.5 & $9.6744 \mathrm{E}-18$ & $2.5533 \mathrm{E}-18$ & $9.6744 \mathrm{E}-18$ & $3.2163 \mathrm{E}-17$ & $9.5745 \mathrm{E}-18$ & $1.9208 \mathrm{E}-18$ \\
\hline 1 & $3.6527 \mathrm{E}-13$ & $6.4114 \mathrm{E}-13$ & $7.6006 \mathrm{E}-13$ & $6.9281 \mathrm{E}-13$ & $4.5593 \mathrm{E}-13$ & $1.0752 \mathrm{E}-13$ \\
\hline 1.5 & $1.5922 \mathrm{E}-10$ & $2.7946 \mathrm{E}-10$ & $3.3128 \mathrm{E}-10$ & $3.0199 \mathrm{E}-10$ & $1.9876 \mathrm{E}-10$ & $4.6868 \mathrm{E}-11$ \\
\hline 2 & $1.1839 \mathrm{E}-08$ & $2.0779 \mathrm{E}-08$ & $2.4632 \mathrm{E}-08$ & $2.2454 \mathrm{E}-08$ & $1.4779 \mathrm{E}-08$ & $3.4848 \mathrm{E}-09$ \\
\hline 2.5 & $3.3374 \mathrm{E}-07$ & $5.8577 \mathrm{E}-07$ & $6.9438 \mathrm{E}-07$ & $6.3299 \mathrm{E}-07$ & $4.1661 \mathrm{E}-07$ & $9.8238 \mathrm{E}-08$ \\
\hline 3 & $5.0911 \mathrm{E}-06$ & $8.9357 \mathrm{E}-06$ & $1.0593 \mathrm{E}-05$ & $9.6559 \mathrm{E}-06$ & $6.3553 \mathrm{E}-06$ & $1.4986 \mathrm{E}-06$ \\
\hline
\end{tabular}

Table 1: Absolute error of our truncated solution with 15 terms.

\begin{tabular}{|c||c|c|c|c|c|c|}
\hline \multicolumn{1}{|c|}{$x$} & 0.5 & 1 & 1.5 & 2 & 2.5 & 3 \\
\hline \hline 0.5 & $2.1676 \mathrm{E}-17$ & $4.3352 \mathrm{E}-17$ & $6.5028 \mathrm{E}-17$ & $8.6704 \mathrm{E}-17$ & $1.0838 \mathrm{E}-16$ & $1.3006 \mathrm{E}-16$ \\
\hline 1 & $2.3843 \mathrm{E}-14$ & $4.7687 \mathrm{E}-14$ & $7.1530 \mathrm{E}-14$ & $9.5373 \mathrm{E}-14$ & $1.1922 \mathrm{E}-13$ & $1.4306 \mathrm{E}-13$ \\
\hline 1.5 & $1.5582 \mathrm{E}-11$ & $3.1164 \mathrm{E}-11$ & $4.6746 \mathrm{E}-11$ & $6.2328 \mathrm{E}-11$ & $7.7910 \mathrm{E}-11$ & $9.3492 \mathrm{E}-11$ \\
\hline 2 & $1.5459 \mathrm{E}-09$ & $3.0918 \mathrm{E}-09$ & $4.6376 \mathrm{E}-09$ & $6.1835 \mathrm{E}-09$ & $7.7294 \mathrm{E}-09$ & $9.2753 \mathrm{E}-09$ \\
\hline 2.5 & $5.4522 \mathrm{E}-08$ & $1.0904 \mathrm{E}-07$ & $1.6357 \mathrm{E}-07$ & $2.1809 \mathrm{E}-07$ & $2.7261 \mathrm{E}-07$ & $3.2713 \mathrm{E}-07$ \\
\hline 3 & $9.9915 \mathrm{E}-07$ & $1.9983 \mathrm{E}-06$ & $2.9975 \mathrm{E}-06$ & $3.9966 \mathrm{E}-06$ & $4.9958 \mathrm{E}-06$ & $5.9949 \mathrm{E}-06$ \\
\hline
\end{tabular}

Table 2: Absolute error of our truncated solution with 15 terms. 


\begin{tabular}{|c||c|c|c|c|c|}
\hline \multicolumn{1}{|c|}{$x^{x}$} & 0.1 & 0.2 & 0.3 & 0.4 & 0.5 \\
\hline \hline 0.1 & $1.3878 \mathrm{E}-17$ & 0 & $1.3878 \mathrm{E}-17$ & 0 & 0 \\
\hline 0.2 & 0 & $1.3878 \mathrm{E}-17$ & 0 & 0 & $2.7756 \mathrm{E}-17$ \\
\hline 0.3 & 0 & $1.3878 \mathrm{E}-17$ & $2.7756 \mathrm{E}-17$ & 0 & $2.7756 \mathrm{E}-17$ \\
\hline 0.4 & $1.3878 \mathrm{E}-17$ & 0 & 0 & $2.7756 \mathrm{E}-17$ & $5.5511 \mathrm{E}-17$ \\
\hline 0.5 & $1.3878 \mathrm{E}-17$ & 0 & $2.7756 \mathrm{E}-17$ & $2.7756 \mathrm{E}-17$ & 0 \\
\hline
\end{tabular}

Table 3: Absolute error of our truncated solution with 15 terms. 